\title{
Laser Resonator Design Using Optical Ray Tracing Software: \\ Comparisons with Simple Analytical Models and Experimental Results
}

\author{
Alexandra Rapaport and Michael Bass \\ School of Optics \\ Center for Research and Education in Optics and Lasers (CREOL) \\ University of Central Florida \\ Orlando, FL 32816-2700
}

Louis S. Weichman

Sandia National Laboratories

Albuquerque, NM
Brian K. Brickeen and Steven W. Green
Honeywell FM\&T
Kansas City, MO

Corresponding author: Michael Bass

Tel.: (407) 823-6977

E-mail: bass@creol.ucf.edu

\begin{abstract}
Software simulation of complex resonator and optical system designs offer the potential to couple traditional opto/mechanical tolerance analysis with the desired system performance. One such software platform, ASAP, and the methodology to perform tolerance analyses has been evaluated and validated for the case of the crossed Porro resonator. Simulation results and experimental measurements are presented and discussed.
\end{abstract}




\title{
Laser Resonator Design Using Optical Ray Tracing Software: \\ Comparisons with Simple Analytical Models and Experimental Results
}

\author{
Alexandra Rapaport and Michael Bass \\ School of Optics \\ Center for Research and Education in Optics and Lasers (CREOL) \\ University of Central Florida \\ Orlando, FL 32816-2700 \\ Louis S. Weichman \\ Sandia National Laboratories \\ Albuquerque, NM \\ Brian K. Brickeen and Steven W. Green \\ Honeywell FM\&T \\ Kansas City, MO \\ Corresponding author: Michael Bass \\ Tel.: (407) 823-6977 \\ E-mail: bass@creol.ucf.edu
}

\section{Introduction}

Lasers have been in use for over 40 years and theories have been developed and verified which describe fairly well their operation. However, with the growing complexity in laser resonator designs and the subsequent increase in the number of intracavity elements, it is often difficult to analytically study and predict resonator 


\section{DISCLAIMER}

This report was prepared as an account of work sponsored by an agency of the United States Government. Neither the United States Government nor any agency thereof, nor any of their employees, make any warranty, express or implied, or assumes any legal liability or responsibility for the accuracy, completeness, or usefulness of any information, apparatus, product, or process disclosed, or represents that its use would not infringe privately owned rights. Reference herein to any specific commercial product, process, or service by trade name, trademark, manufacturer, or otherwise does not necessarily constitute or imply its endorsement, recommendation, or favoring by the United States Government or any agency thereof. The views and opinions of authors expressed herein do not necessarily state or reflect those of the United States Government or any agency thereof. 


\section{DISCLAIMER}

Portions of this document may be illegible in electronic image products. Images are produced from the best available original document. 
performance. Laser engineers have therefore had to rely on experience and empirical $\mathbf{S T}$ methods to build, improve, and optimize complex resonators. Today, lasers have a major role in several high technology areas and mass production of highly reliable and predictable devices is necessary. It is therefore necessary for laser engineers to be able to fully understand the function of their system before building expensive prototypes. Different commercially available software programs have been used to analyze various resonators (Paraxia, ). We suggest and demonstrate the use of ASAP $^{*}$ as a valuable tool to analyze the specific cases of the crossed Porro and corner cube based laser cavities. Initial validation of the proposed ASAP solution methodology is obtained by comparing the model results for a simple Porro prism ended cavity against the closed-form analytical solutions previously published (Ref. [2] to [4]). We then apply the same ASAP solution routines to a more complex cavity and present experimental confirmation of the modeled behavior.

\section{ASAP model comparison to earlier published analytical solutions for the case of the simple laser resonator}

To verify the validity of our use of ASAP for modeling laser resonators, we first used the software to corroborate the theoretical results established in Ref. [2]. The case under consideration is a simple crossed Porro cavity in which the crests of the prisms are oriented at an azimuth angle $\theta$ to each other. We include a gain medium of refractive index $n=1.82$ (e.g., YAG) and a polarizing cube beam splitter to provide an output for the laser energy. The appropriate dimensions and angles are shown in Fig. 1. The Porro

\footnotetext{
* ASAP is an optical programming language and is produced by Breault Research Organization, Inc.[1]
} 
prism on the right (Prism 2) has its crest located horizontally (along the X-axis), while the ridge of the prism on the left (Prism 1) is rotated about the Z-axis to make an angle $\theta$ with respect to the $\mathrm{X}$-axis.

Closed-form analytical solutions are provided in Ref. [2] and [3] for the displacement and deviation angle of the laser oscillation axis that results from the introduction of a misalignment angle $\beta$ imposed on Porro prism 2. The authors determine the laser performance parameters listed above as a function of the Prism's azimuth angle $\theta$ and the various resonator lengths defined in Fig. 1. (It should be noted that in the development of the closed-form analytical solutions given in Ref [2] and [3] only one of the end Porro prism is perturbed - all other intracavity components are assumed to be stationary and perfectly aligned to the unperturbed oscillation axis. Any additional linear or angular misalignment of these other intracavity elements would result in a much more complicated closed-form analytical solution - suggesting a severe limitation on the applicability of this approach to a more complex tolerance analysis.) We first used the iteration macro capability in ASAP to design a subroutine that identifies the laser cscillation axis. Determination of the oscillation axis and the resultant direction cosines are defined by a ray that when propagated within the resonator returns parallel to itself after several roundtrips. Once the direction of this axis is determined, we can obtain its position at a distance "l" from the beam splitter directly from the output window function in ASAP.

The command script written for ASAP defines the optical system as described in Fig. 1. Various subroutines and macros then initiate a grid of rays that oscillate within the resonator given the specified misalignment tilts for Porro prism 2 (i.e. angular tilts $\alpha_{\mathrm{X}}$ 
about the $\mathrm{X}$-axis and $\alpha_{\mathrm{Y}}$ about the $\mathrm{Y}$-axis). The direction of those rays located at the face of the rod closest to the beam splitter is calculated and recorded after a single roundtrip and then again after 13 roundtrips. (The choice of 13 roundtrips is arbitrary. However, the number of roundtrips must be sufficient to verify that the bundle of rays oscillating within the resonator are stable and yet not prove too time consuming in deriving the solution.) We define a quality factor as the difference between the ray's direction after a single roundtrip and again after 13 roundtrips. The iteration function in ASAP (Appendix A, macro command \$TER) increases in a stepwise fashion the angles $\alpha_{X}$ and $\alpha_{Y}$ at which the ray bundles are launched and returns an output value for the quality factor for each set of angles. At the end of the model run ASAP determines the set of ray angles that minimizes the output value of the quality factor.

For tilts about the $\mathrm{X}$-axis (or vertical misalignment) imposed on Porro prism 2 the direction of the optical axis is found to be unchanged for all azimuth angles $\theta-$ as predicted and observed in Ref. [2]. Similarly, the output beam is not displaced nor is the energy affected by the small vertical misalignments imposed. (It should be noted that this is valid for those tilts or rotations of Porro prism 2 that do not exceed the total internal reflection angle at the back face of the prism). In the remainder of the paper, we focus on those more interesting conditions that result from tilting the Porro prism about its sensitive axis (Y-axis or horizontal misalignment).

The results obtained for several sets of azimuth and horizontal misalignment angles are tabulated in Table 1. The values are compared to those results given by the analytical solutions described in Ref. [2], which were subsequently shown to agree with the collected experimental data. The direction of the optical axis given by ASAP is found 
to be in complete agreement with the analytical model developed by Lee and therefore also accurately describes the experimental observations that he reported.

In order to calculate the displacement of the beam caused by the horizontal maisalignment of Porro prism 2 in Fig 1, a grid of rays is launched parallel to the direction of the optical axis as discussed earlier. The rays are then traced through the optical system until they reach a detector or thermal paper located at a distance " $d$ " from the cube beam splitter. The position of the beam centroid in the plane of the detector is read from the ASAP output window after each of 6 roundtrips and the average of 6 of these sets are then calculated. The average position is obtained for several different azimuth angles of the front prism (Prism 1 in Fig. 1) when the cavity is perfectly aligned as well as for several misalignment angles of the back prism (Prism 2). The value calculated for the perfect cavity is then subtracted from the results for the various misalignments and the cutcome is compared to the analytical solution developed in Ref. [3]. In that paper the climension of the polarizing cube is not specified, so we set the length of each side to 2 $\mathrm{cm}$. For this size beam splitter, the displacement of the beam at the exit face of the output polarizer calculated by ASAP is about $10 \%$ less than the value given by the theory. However, because the beam is now pointing in a tilted direction about both the $\mathrm{X}$ and $\mathrm{Y}$ axes, it is observed that we can obtain those same results as given by the experiment when we translate the detector plane away from the output polarizer by $1.5 \mathrm{~cm}$. This distance between the thermal paper and the beam splitter seems reasonable. At $1.5 \mathrm{~cm}$ the paper is very near the exit face but the material ejected by the ablation will not deposit on the optics as it would if the thermal paper were too close. It is therefore probable that the experimental data taken to confirm the theory in Ref. [3] were measured at a small 
distance from the cube. The displacements calculated by ASAP are a perfect fit to the reported experimental data if the burns were made $1.5 \mathrm{~cm}$ from the output face of the beam splitting cube (see Fig. 2). (A more thorough experimental verification of the angular change in the laser oscillation axis and the resultant displacements in the output plane were carried out as part of this work on a second breadboard laser system available in our laboratory).

Finally, the mode volume within the gain medium was calculated using two different methods for each set of tilt and horizontal misalignment angles. First, as described in Ref. [4] the position of the optical axis was calculated in the rod. The laser rod, being the smallest aperture in the optical system, effectively determines the beam diameter within the resonator. Hence, the smallest radial distance between the optical axis and the edge of the rod dictates the mode volume in the active medium - in this case assumed to be cylindrical. We read the position of the optical axis on the rod directly from the ASAP output window function. Subsequently, using the reported co-ordinates for the beam centroid $\left(x_{0}\right.$ and $\left.y_{0}\right)$ locating the ray bundle on the rod face, we calculate the resultant mode volume and normalize this value to the volume expected in the case of a perfectly aligned cavity. (This effectively yields the ratio of the output energies under the misaligned and perfectly aligned conditions). In Ref. [4] this ratio is given as:

$$
\frac{\mathrm{E}(\beta)}{\mathrm{E}\left(0^{\circ}\right)}=\frac{\pi\left(\mathrm{a}-\sqrt{\Delta \mathrm{x}_{0}^{2}+\Delta \mathrm{y}_{0}^{2}}\right)^{2}}{\pi \mathrm{a}^{2}}
$$


Here, $\mathrm{E}(\beta)$ and $\mathrm{E}\left(0^{\circ}\right)$ are the output energies of a cavity in which the back prism presents a misalignment angle $\beta$ and that of the perfectly aligned cavity $\left(\beta=0^{\circ}\right)$, "a" is the radius of the laser rod, and $\Delta x_{0}$ and $\Delta y_{0}$ are the difference between the centroid coordinates for a tilt of $\beta$ and that of the perfectly aligned cavity.

The results of our ASAP calculations (plain circles) are plotted in Fig. 3 along with the analytical results previously published (dashed curves) for 3 different azimuth angles $\theta(30,55$ and 60 deg.). Once again, close agreement validates the ASAP results and assumptions.

A second method was then used to evaluate the mode volume inside the rod. For each set of tilt and misalignment angles, a grid of rays filling the whole surface of the rod was launched along the direction of the optical axis. After several roundtrips, the number of rays left in the resonator was read from the ASAP output window. This number was normalized to the number of rays left in a perfectly aligned cavity and plotted in Fig. 3 with a cross. For most tilt angles the analytical results and those calculated using ASAP are very close (within 3-4\%) as seen in Fig. 3. Indeed, for most tilt angles the rays do not close on themselves and after a few roundtrips the beam is regularly reshaped - acquiring a circular cross-section (Fig. 4a). However, for azimuth angles that are integral divisors of 90 degrees the beam acquires a very clear, non-circular symmetry (see Fig. 4b). In this case, the assumption that the mode volume is a cylinder comprised of a radius given by the distance between the optical axis and the edge of the laser rod is no longer accurate. We observe discrepancies of up to $10 \%$ between the 2 methods (shown in Fig. 3 for a tilt angle of 60 degree). This could explain the smaller than predicted slope that the 
experimental data of Ref. [4] seems to suggest for misalignment angles less than 10 arc $\min$.

Finally, a subroutine was written that identified the value of the horizontal misalignment angle $\beta$ at which the output energy was half that of the perfectly aligned cavity - iterated over a range of azimuth angles. Fig. 5 shows the results obtained by ASAP (plain circle) compared against the analytical results of Ref. [4] for the case in which the refractive index of the rod was taken into account (dashed curve). It is clear that the ASAP simulation accurately predicts the performance of the simple cavity studied here over a wide range of tilt and misalignment angles. Moreover, unlike the closed-form analytical solutions, given the proper choice of modeling software and the appropriate subroutines, the ease of obtaining the required parametric solutions does not increase with the number, complexity, and/or the initial positions of the intracavity elements.

\section{Application of ASAP modeling to a more complex cavity}

It was shown for the simple laser cavity that the ASAP model is an accurate tool for the study and analysis of laser resonators containing prisms. In this section we expanded the ASAP modeling and verification activities to a cavity containing a greater number of optical components as shown in Fig. 6. In system applications where precise control of beam position under dynamic environments (i.e. vibration, shock, temperature, etc.) is crucial an understanding of the effects of component misalignments on the lasers ultimate performance is essential. Furthermore, mass manufacturing of the laser and beam delivery systems require a thorough optical and mechanical tolerance analysis of intracavity elements to establish acceptable component parameters in an effort to properly 
balance alignment efforts, performance, and producability against per unit cost. As will be demonstrated, the ASAP program is one potential software tool that could be utilized to further aid the optical designer in the complex process of establishing component specifications and determining system performance under dynamic environments. In this section we present further validation activities of the ASAP software directed towards such an application. The initial validation process described herein includes: 1) assembly and characterization of a "breadboard" laser cavity representative of the ultimate system application, 2) model simulation describing the interaction of all intracavity optical elements, and 3) comparison of experimental data to simulation results for various intracavity component misalignments.

A complete set of component misalignment conditions can be constructed based on the superposition of linear displacements and angular perturbations imposed on the selected intracavity element(s). Furthermore it is assumed that the combined effects induced by any combination of component displacement and angular misalignment displays a linear functional dependence. Based on this assumption we have considered the misalignment conditions separable and have performed our experimental and verification activities in a like manner - evaluating linear component displacements followed by angular perturbations of the intracavity elements.

First, the effect of beam position and output energy for component linear displacements without angular tilt was studied. Each alignment critical component (Porro prisms and laser rod) within the laser breadboard shown in Fig. 6a were linearly displaced and the resultant output energy was recorded. Similarly, the corresponding elements within the ASAP model were subjected to the same displacements and the resultant 
output beam displacement and output energy was calculated. (See Fig. $6 \mathrm{~b}$ for experimental setup and associated target planes for measured and calculated beam displacements). It is easily confirmed that the pointing direction of the laser oscillation axis does not vary with linear displacement of the various intracavity components. However, the laser oscillation axis is linearly displaced within the laser cavity and subsequently the intracavity beam becomes apertured by the laser rod. This effectively produces additional cavity losses and a corresponding reduction of the output energy.

Beam displacements at the target plane on $\mathrm{CCD}$ camera 2 were calculated using the ASAP beam centroid as described earlier for the simple resonator. Also recorded in the resultant data set are the number of rays that remain stable in the resonator cavity after the specified number of round-trips. This number is directly related to the mode volume inside the gain medium, hence reflecting the change in output energy of the laser. Normalizing the calculated mode volume (or energy) against the case of the perfectly aligned cavity then provides an estimate of the effective change in laser performance due to the subjected misalignment.

The results of the ASAP simulation describing the displacement of the output beam against linear misalignment along the $\mathrm{X}$ and $\mathrm{Y}$ axes for Porro prism 1, Porro prism 2, and the laser rod are plotted in Figs. 7a through 9a. Similarly, the calculated normalized energy output versus those same misalignments are plotted in Figs. 7b through $9 \mathrm{~b}$ along with the experimental data for comparison. (The displacements of the output beam at CCD camera 2 were not measured experimentally for component linear misalignments). 
As can be seen from Figs. $7 b, 8 b$ and $9 b$ for the normalized output energies, the agreement between the ASAP simulation with the experimental data is extremely good. From this data we conclude that a linear misalignment along the $\mathrm{X}$ axis for Porro prism 1 produces a noticeable displacement of the output beam and introduces a corresponding loss in the output beam energy. In contrast, a misalignment along the $\mathrm{Y}$-axis has no effect on the beam position or output energy (Figs. 7a and b) - until the edge of the prism effectively acts as the defining intracavity aperture. We also observe that displacing Porro prism 2 along any direction does not displace the beam in the horizontal (XZ) plane (Fig. $8 \mathrm{a}$ and $b$ ). Similarly, a linear displacement of the laser rod does not produce a displacement of the output beam. However because the rod is the defining intracavity aperture, linear displacement results in clipping of the beam and the system output energy is proportionately reduced (Figs. 9a and b).

We then studied the effect of angular misalignment of the end prisms on the performance of the laser. Determination of the laser oscillation axis for each misalignment angle was accomplished using the methodology described earlier for the case of the simple cavity. As shown in Fig. 10, substantial tilts or rotations $\left(+/-2^{\circ}\right)$ about the $\mathrm{Y}$-axis of Porro prism \#1 produces no change in the pointing direction of the output beam and little change in the laser output energy. (It is this insensitivity to tilt along the prism crest that makes the crossed Porro laser cavity attractive for applications in harsh environments). However, if the tilt/rotation becomes too large the angle of incidence on the inside surface of the prism exceeds the angle for total internal reflection (TIR) and lasing decreases abruptly. Even under loss of TIR it is possible to use ASAP to calculate and simulate the effective reduction in the Fresnel coefficient of reflection. We observe 
that the number of stable rays in the cavity multiplied by the cube of the coefficient of reflection measured at the prism backface accurately fits the experimental data collected. It is interesting to note that the pulse width obtained with the experimental setup is about 15 ns.. Given the physical dimensions of the breadboard, this pulse width roughly equates to three cavity roundtrips - or three reflections at the prism back surface. (Hence the cube factor for the coefficient of reflection). However other factors (like the gain of the active medium) should influence the output energy and pulse length so a more complete study involving other cases is needed before we can draw a stronger conclusion.

Once the direction of the optical axis was established, it was straightforward to study the effect of a selected component misalignment angle on the beam position at the target plane (CCD camera 2) and the output energy as it was done for the case of linear displacements. A comparison of the ASAP simulation to the experimental results for each tilt axis of Porro prism 2 is provided in Fig. 11a and b. Again, simulation and the experiment provide excellent agreement. We also note that any tilt of Porro prism 2 correspondingly decrease the laser output energy, however, more importantly the tilt imparted will strongly effect the degree of beam wander - potentially steering the beam from its intended target.

The methodology presented herein for alignment sensitivity using ASAP is not limited to the study of a single component parameter or resonator configuration. It can quickly be implemented to look at the effect of any combination of various misalignments without significant complications - unlike closed-form analytical or graphical solutions that must be extensively modified to reflect the desired geometry. 


\section{Assumptions, Extension to Other Resonator Configurations, and Future Activities}

In developing the simulation methodology using ASAP a number of assumptions have been made or implied. Specifically, in the case of the crossed Porro resonators described and modeled herein it was assumed that the intracavity and subsequent output intensity spatial profiles were uniform over the beam cross sectional area. This assumption yields the relatively simple expression for the change in output energy as a function of the intracavity beam diameter (or cross sectional area) as expressed in Eq. 1 and Ref.[4]. In addition to uniform intensity, the current ASAP simulation contained no active gain element (rod gain $=1$ ) nor modeled other traditional "physical" optic effects (i.e. polarization, diffraction, phase front aberrations, etc.). However, it should be noted that these assumptions - appropriate for the crossed Porro resonator - should not be viewed as an inherent limitation of the ASAP simulation.

Further model refinements and application of the ASAP software to other resonator configurations are currently under investigation. Future plans include: 1) coupling the relevant laser equations with the modeled resonator parameters (i.e. intracavity loss, output polarization coupling, temperature variation of laser cross section, etc.) to better reflect those effects on laser performance, 2) extending the model methodology and subsequent verification to other resonator configurations characterized by single and/or mutimode longitudinal spatial profiles, 3) demonstrating automated optimization routines for tolerance analysis of selected intracavity components, and 4) coupling the optical source code with a mechanical analysis package to provide a single platform for parametric study under simulated environmental conditions. 


\section{Conclusion}

A computational method using commercially available optical ray tracing software, ASAP, has been shown to accurately represent the performance of the crossed Porro prism resonator. This conclusion was demonstrated by comparing the results calculated with ASAP to the "closed-form" analytical solutions for the simple Porro prism ended resonator and by similar comparison to experimental data collected for both the simple and a more complex resonator designs. We also demonstrated the reshaping of the beam that will occur if the azimuth angle between the Porro prism crests is an integer divisor of 90 degrees. This would not be revealed in the analytical model. The simulation techniques developed to determine the resultant laser oscillation angle and output energy under intracavity misalignments can easily be applied to more complex resonator configurations. It is an extremely valuable tool for analyzing cavities without having to build expensive prototypes.

\section{Disclaimer}

All data prepared, analyzed, and presented has been developed in a specific context of work and was prepared for internal evaluation and use pursuant to that work authorized under the referenced contract. Reference herein to any specific commercial product, process or service by trade name, trademark, manufacturer, or otherwise, does not necessarily constitute or imply its endorsement, recommendation, or favoring by the United States Government or any agency thereof, or Honeywell International Inc. 


\section{Acknowledgements}

Funding of this work by Sandia National Laboratories and the United States Department of Energy Contract No. DE-ACO4-76DP00613 and Honeywell Inc. is gratefully acknowledged. Sandia is a multiprogram laboratory operated by Lockhead Martin for the United States Department of Energy under Contract DE-AC04-

94AL85000. We also acknowledge the donation of ASAP software by Breault Research Organization, Inc. 


\section{Appendix A}

\section{Description of the flowchart in Fig. 12:}

The left part of the flowchart is executed first. It determines the direction of the optical axis for various misalignments (linear displacement, angular tilt, one or several elements of the cavity over the range for which one wants to study the tolerancing). The flowchart of Fig. 12 shows as an example a tilt of one of the Porro prisms. First, an optical system in which a tilt has been introduced is defined. The \$ITER function predefined in ASAP is then called in which we define 2 angles $\left(\alpha_{X}\right.$ and $\left.\alpha_{Y}\right)$ for the direction of the source of rays. The program then traces the rays and the direction of the grid of rays is measured at one surface (e.g., face of the laser rod) after 1 round trip and then again after 13 roundtrips (sufficient to insure enough stability of the beam inside the cavity). The difference is calculated and transferred as an output to \$ITER. The macro command \$ITER then steps through the possible values of $\alpha_{Y}$ keeping $\alpha_{X}$ constant. It then increments $\alpha_{X}$ and performs the calculation again. At the end of the execution of \$ITER, the value of $\left(\alpha_{X}, \alpha_{Y}\right)$ that minimizes the difference (makes it zero if the optical axis lies within the range) is returned in the output window and can be read to serve as an input to the right part of the loop.

The right part of the loop looks at the laser performance as the misalignment is increased. Inside a \$DO loop that covers the range of tolerancing that one wants to study, the tilt angle (or linear displacement) is determined. The optical system is then defined with this tilt angle, and a set of rays parallel to the calculated optical axis direction (determined in the left part of the flowchart) is defined. They are then traced through the system for 18 roundtrips to ensure resonator stability. The \$DO loop has as output the 
number of rays inside the cavity, the dimensions of the beam, the coefficient of reflection of the Porro prisms (when tilted, it is possible to exceed the condition for total internal reflection and so the Fresnel reflection coefficients are calculated in ASAP), and the relative flux. The results are finally normalized to the values for the perfectly aligned cavity. 


\section{References}

1] ASAP is a non-sequential ray-tracing program that is developed by Breault Research Organization, Inc. to give a powerful set of basic optical analysis tools to allow the user to perform complicated optical analyses. Some of these tools are: unconstrained 3D entity modeling, macro programming language, and Gaussian beam propagation algorithm. 2] Jyh-Fa Lee and Chung-Yee Leung, "Beam pointing direction changes in a misaligned Porro prism resonator", Applied Optics 27, No. 13, 2701-2707 (1988)

3] Jyh-Fa Lee and Chung-Yee Leung, "Lateral displacement of the mode axis in a misaligned Porro prism resonator", Applied Optics 28, No. 24, 5278-5284 (1989) 4] Jyh-Fa Lee and Chung-Yee Leung, "Method of calculating the alignment tolerance of a Porro prism resonator", Applied Optics 28, No. 17, 3691-3697 (1989) 
Table 1:

Tilt Horizontal $\alpha_{x}$ in $\alpha_{y}$ in Output beam Output beam angle misalignment misaligned misaligned direction about direction about (deg)

30

\begin{tabular}{|c|c|c|c|c|c|c|c|}
\hline \multirow[t]{2}{*}{ (deg) } & \multirow{2}{*}{$\begin{array}{c}\text { angle } \\
\text { (Arc min) }\end{array}$} & \multirow{2}{*}{$\begin{array}{c}\text { prism } \\
\text { (Arc min) }\end{array}$} & \multirow{2}{*}{$\begin{array}{c}\text { prism } \\
\text { (Arc min) }\end{array}$} & \multicolumn{2}{|c|}{ Y-axis (Arc min) } & \multicolumn{2}{|c|}{ Z-axis (Arc min) } \\
\hline & & & & ASAP & $\begin{array}{l}\text { Analytical } \\
\text { solution }\end{array}$ & ASAP & $\begin{array}{c}\text { Analytical } \\
\text { solution }\end{array}$ \\
\hline & -5 & -0.0833 & -1.916 & 5 & 5 & -2.8867 & -2.8868 \\
\hline & -4 & -0.0667 & -1.533 & 4 & 4 & -2.3094 & -2.3094 \\
\hline & -3 & -0.05 & -1.150 & 3 & 3 & -1.7321 & -1.7321 \\
\hline & -2 & -0.0333 & -0.766 & 2 & 2 & -1.1548 & -1.1547 \\
\hline & -1 & -0.0167 & -0.383 & 1 & 1 & -0.5772 & -0.5774 \\
\hline \multirow[t]{11}{*}{30} & 0 & 0 & 0 & 0 & 0 & 0.0000 & 0.0000 \\
\hline & 1 & 0.0167 & 0.383 & -1 & -1 & 0.5772 & 0.5774 \\
\hline & 2 & 0.0333 & 0.766 & -2 & -2 & 1.1547 & 1.1547 \\
\hline & 3 & 0.05 & 1.150 & -3 & -3 & 1.7321 & 1.7321 \\
\hline & 4 & 0.0667 & 1.533 & -4 & -4 & 2.3094 & 2.3094 \\
\hline & 5 & 0.0833 & 1.916 & -5 & -5 & 2.8867 & 2.8868 \\
\hline & -5 & -0.0833 & -1.916 & 5 & 5 & -5.0006 & -5.0000 \\
\hline & -4 & -0.0667 & -1.533 & 4 & 4 & -4.0002 & -4.0000 \\
\hline & -3 & -0.05 & -1.150 & 3 & 3 & -3.0003 & -3.0000 \\
\hline & -2 & -0.0333 & -0.766 & 2 & 2 & -2.0000 & -2.0000 \\
\hline & -1 & -0.0167 & -0.383 & 1 & 1 & -1.0003 & -1.0000 \\
\hline \multirow[t]{6}{*}{45} & 0 & 0 & 0 & 0 & 0 & 0.0000 & 0.0000 \\
\hline & 1 & 0.0167 & 0.383 & -1 & -1 & 1.0005 & 1.0000 \\
\hline & 2 & 0.0333 & 0.766 & -2 & -2 & 2.0002 & 2.0000 \\
\hline & 3 & 0.05 & 1.150 & -3 & -3 & 3.0005 & 3.0000 \\
\hline & 4 & 0.0667 & 1.533 & -4 & -4 & 4.0000 & 4.0000 \\
\hline & 5 & 0.0833 & 1.916 & -5 & -5 & 5.0000 & 5.0000 \\
\hline
\end{tabular}

45 


\section{Figure captions}

Figure 1: Schematic of the simple 2 Porro-prism cavity studied analytically in the literature and used for validation of the ASAP modeling.

Figure 2: Lateral displacement of the output beam versus horizontal misalignment angle of Porro prism 2 in Fig. 1 for various azimuth angles of Porro prism 1. The hollow symbols represent the displacement at $1 \mathrm{~cm}$ along the optical axis measured from the output coupling beam splitter. The solid symbols represent the displacement at 2.5

$\mathrm{cm}$. The triangles are the results of the simulation for $\theta=80 \mathrm{deg}$, the squares are for $\theta=70 \mathrm{deg}$ and the circles are for $\theta=60 \mathrm{deg}$. The lines represent the results of the analytical model for the same azimuth angles, when the refractive index of the laser rod is taken into account (see Ref. [3])

Figure 3: Output energy normalized to the energy of a perfectly aligned cavity versus the horizontal misalignment angle of Porro prism 2 for different azimuth angles of Porro prism 1: $\theta=30 \mathrm{deg}, \theta=55 \mathrm{deg}$ and $\theta=60 \mathrm{deg}$. The plain spheres represent the outcome of the ASAP simulation when the energy is considered proportional to the square of the distance between the optical axis and the edge of the laser rod. The curves represent the analytical results of Ref. [4] for the same azimuth angles (case in which the refractive index of the laser rod is taken into account). The crosses represent the outcome of the ASAP simulation when the number of rays left in the cavity after several roundtrips is considered to be a measure of the energy.

Figure 4: Plot of the profile of the beam oscillating in the cavity as seen on one face of the rod.

a) azimuth angle of $55 \mathrm{deg}$ 
b) azimuth angle of $60 \mathrm{deg}$

Figure 5: Horizontal misalignment angle of Porro prism 2 at which the output energy is half the output energy of the aligned cavity versus azimuth angle of Porro prism 1. The filled circles are the results given by the ASAP simulation whereas the curve is given by the theory in Ref. [4] when the refractive index of the laser rod is taken into account.

Figure 6: Layout of the more complex breadboard cavity studied.

a) Position of the various elements of the cavity.

b) Experimental set-up for measuring displacements and energies.

Figure 7: Effect of a displacement of Porro prism 1 in Fig. 6a on the output beam.

a) ASAP simulation of the linear displacement of the output beam measured on the CCD 2 in Fig. $6 \mathrm{~b}$ due to a misalignment of Porro prism 1. The solid lines correspond to a misalignment of Porro prism 1 along the $\mathrm{X}$-axis, the dotted lines correspond to a misalignment along the $\mathrm{Y}$-axis. The horizontal bars represent a displacement of the beam in the horizontal direction (XZ plane), the vertical bars represent a displacement in the $\mathrm{Y}$ direction.

b) Energy of the output beam normalized to the energy of a perfectly aligned cavity. The diamond shapes are experimental data points, the lines represent the calculations from ASAP. The solid symbols and line correspond to a misalignment along the $\mathrm{X}$-axis and the hollow symbols and dotted line correspond to a misalignment along the $\mathrm{Y}$-axis.

Figure 8: Effect of a displacement of Porro prism 2 in Fig. 6a on the output beam. 
a) ASAP simulation of the linear displacement of the output beam measured on the CCD 2 in Fig. $6 \mathrm{~b}$ due to a misalignment of Porro prism 2 . The solid lines correspond to a misalignment of Porro prism 2 along the $\mathrm{X}$-axis, the dotted lines correspond to a misalignment along the $\mathrm{Y}$-axis. The horizontal bars represent a displacement of the beam in the horizontal direction (XZ plane), the vertical bars represent a displacement in the $\mathrm{Y}$ direction.

b) Energy of the output beam normalized to the energy of a perfectly aligned cavity. The diamond shapes are experimental data points, the lines represent the calculations from ASAP. The solid symbols and line correspond to a misalignment along the $\mathrm{X}$-axis and the dotted line corresponds to a misalignment along the $\mathrm{Y}$-axis. Figure 9: Effect of a displacement of the laser rod on the output beam.

a) ASAP simulation of the linear displacement of the output beam measured on the CCD 2 in Fig. $6 \mathrm{~b}$ due to a misalignment of the laser rod. The solid lines correspond to a misalignment of the laser rod along the $\mathrm{X}$-axis, the dotted lines correspond to a misalignment along the $\mathrm{Y}$-axis. The horizontal bars represent a displacement of the beam in the horizontal direction ( $\mathrm{XZ}$ plane), the vertical bars represent a displacement in the $\mathrm{Y}$ direction.

b) Energy of the output beam normalized to the energy of a perfectly aligned cavity. The diamond shapes are experimental data points, the line represents the calculations from ASAP (same result obtained for a misalignment along the $\mathrm{Y}$-axis as for the $\mathrm{X}$-axis). The solid symbols correspond to a misalignment along the $\mathrm{X}$-axis and the hollow symbols correspond to a misalignment along the $\mathrm{Y}$-axis. 
Figure 10: Effect of an angular misalignment of Porro prism 1 in Fig. 6a on the output beam.

a) Linear displacement of the output beam measured on the CCD 2 in Fig. $6 \mathrm{~b}$ due to a tilt of Porro prism \#1 about the X-axis. The symbols are experimental data points. The diamonds correspond to a horizontal displacement (XZ plane), the spheres correspond to a vertical displacement (along the Y-axis). The solid lines are the result of the simulation using ASAP. The horizontal bars correspond to a horizontal displacement ( $\mathrm{XZ}$ plane), the vertical bars correspond to a vertical displacement (along Y-axis).

b) Energy of the output beam normalized to the energy of a perfectly aligned cavity. The diamond shapes are experimental data points and the lines represent the calculations from ASAP. The solid symbols and line correspond to a tilt about the Xaxis and the hollow symbols and dotted line correspond to a tilt about the $\mathrm{Y}$-axis.

Figure 11: Effect of an angular misalignment of Porro prism 2 in Fig. 6a on the output beam.

a) Linear displacement of the output beam measured on the CCD 2 in Fig. $6 b$ due to a tilt of Porro prism 2 in Fig. 6 a about the Y-axis. The symbols are experimental data points. The diamonds correspond to a horizontal displacement (XZ plane), the spheres correspond to a vertical displacement (along the Y-axis). The dotted lines are the result of the simulation using ASAP. The horizontal bars correspond to a horizontal displacement (XZ plane), the vertical bars correspond to a vertical displacement (along Y-axis). 
b) Energy of the output beam normalized to the energy of a perfectly aligned cavity. The diamond shapes are experimental data points and the lines represent the calculations from ASAP. The solid symbols and line correspond to a tilt about the Xaxis and the hollow symbols and dotted line correspond to a tilt about the $\mathrm{Y}$-axis.

Figure 12: Flowchart of the automated process in ASAP as discussed in the appendix. 


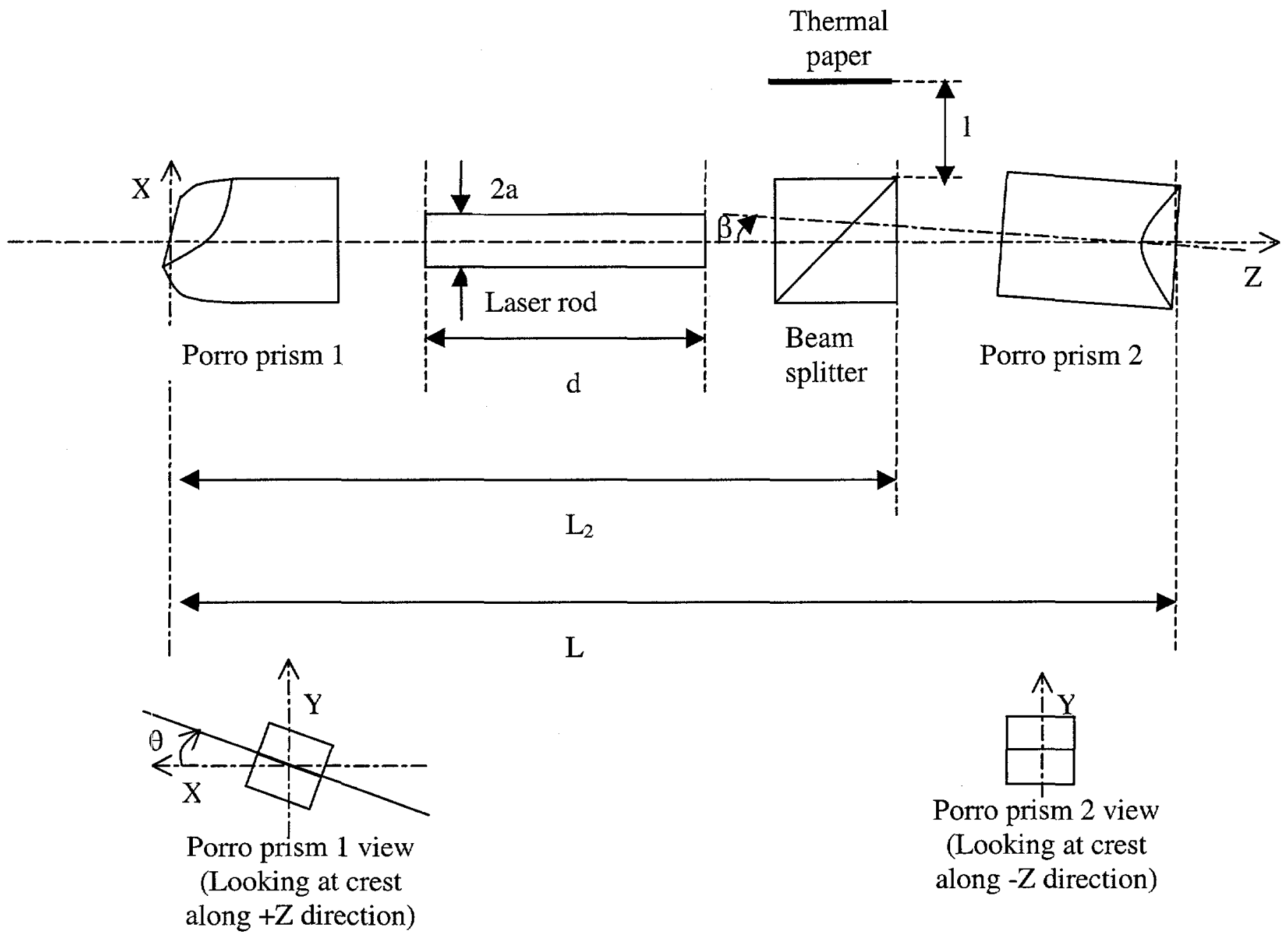

Figure 1 


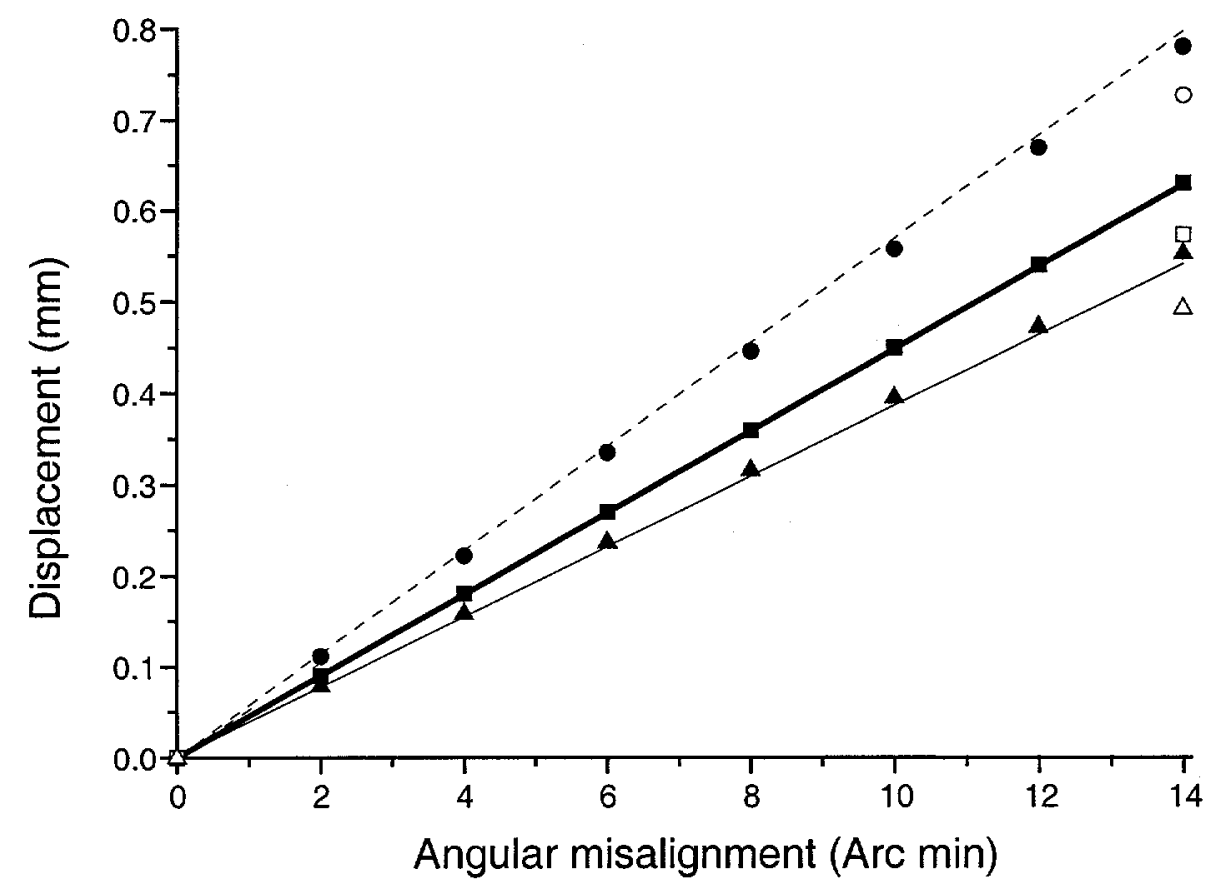

Figure 2 


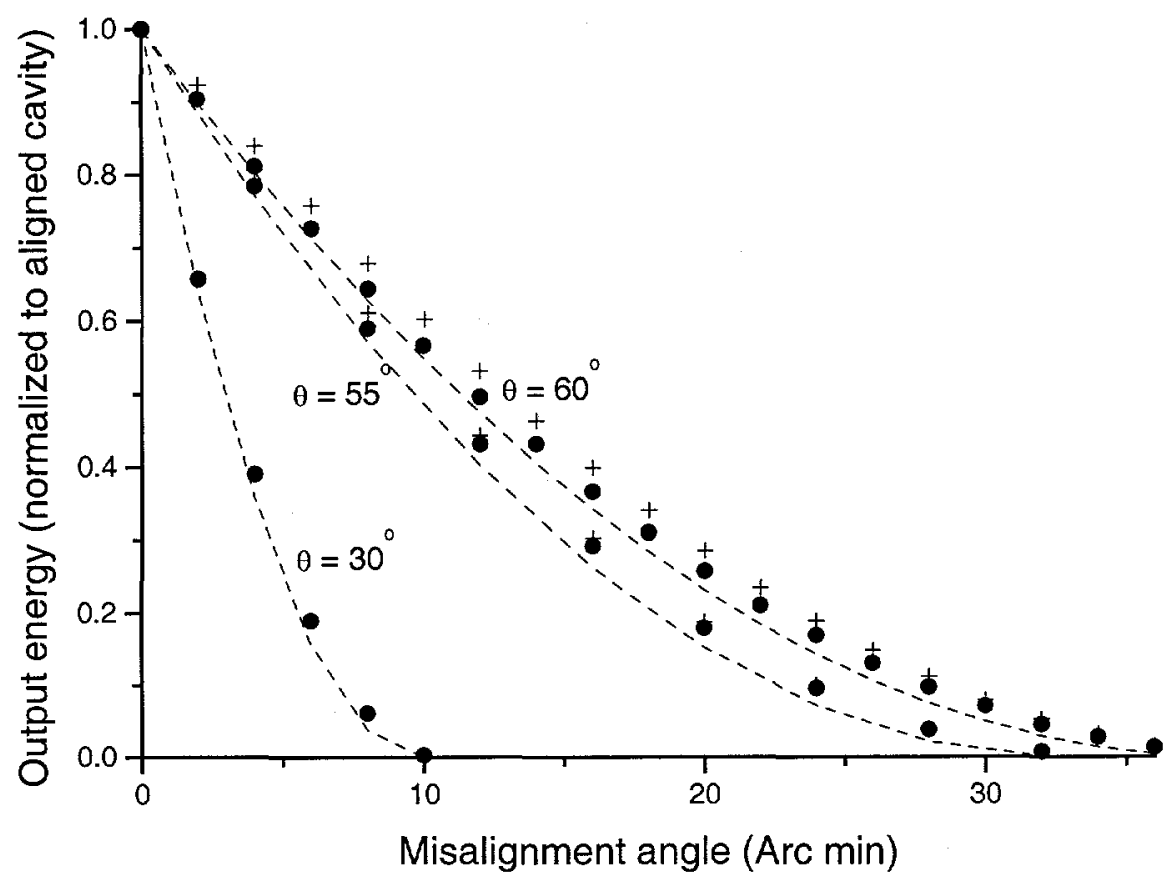

Figure 3 
a)

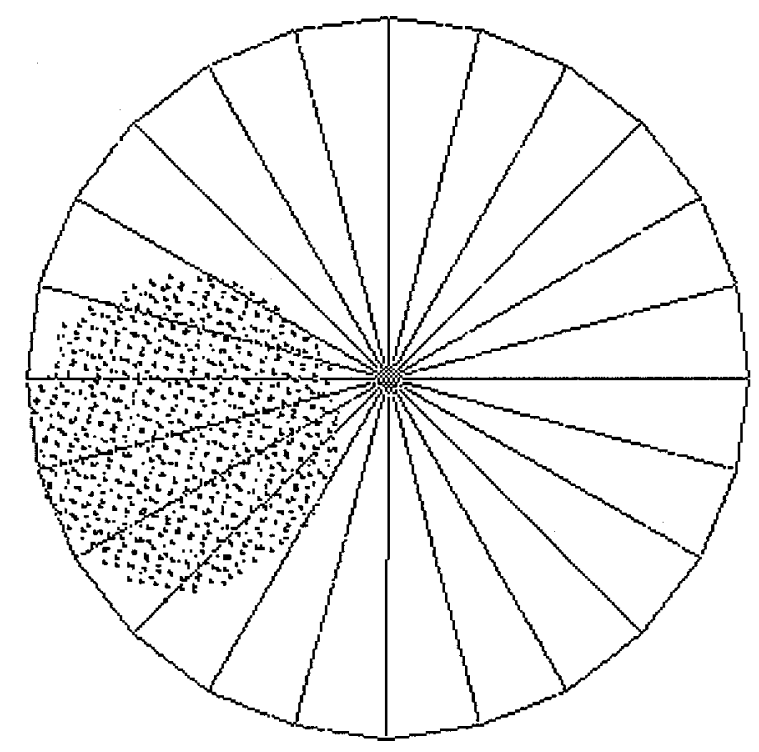

b)

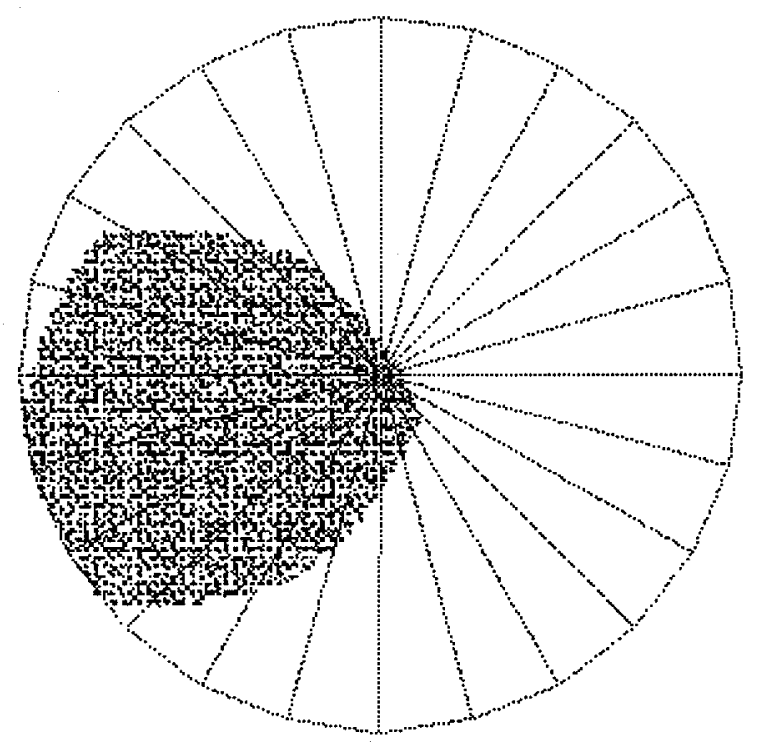

Figure 4 


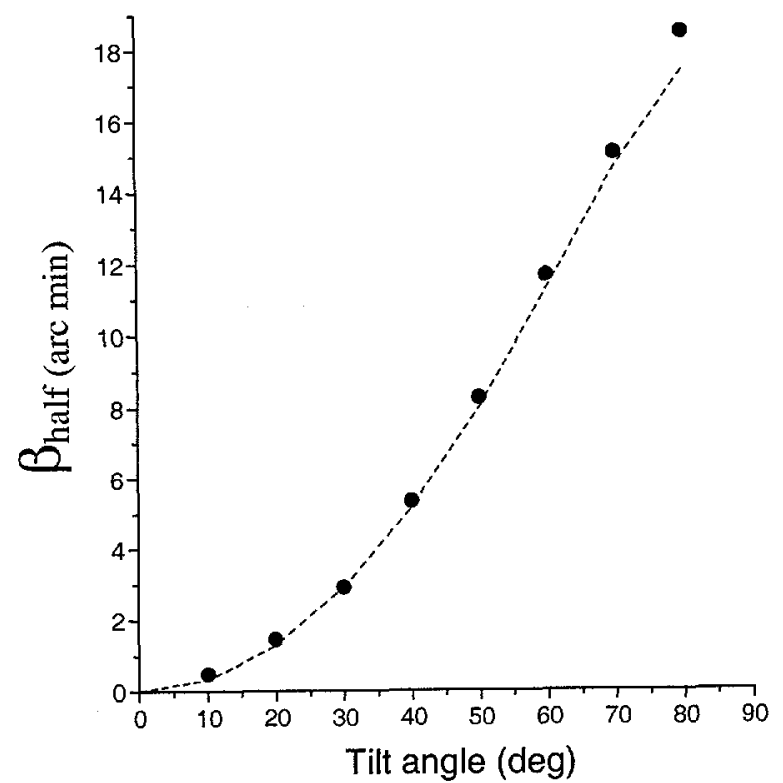

Figure 5 


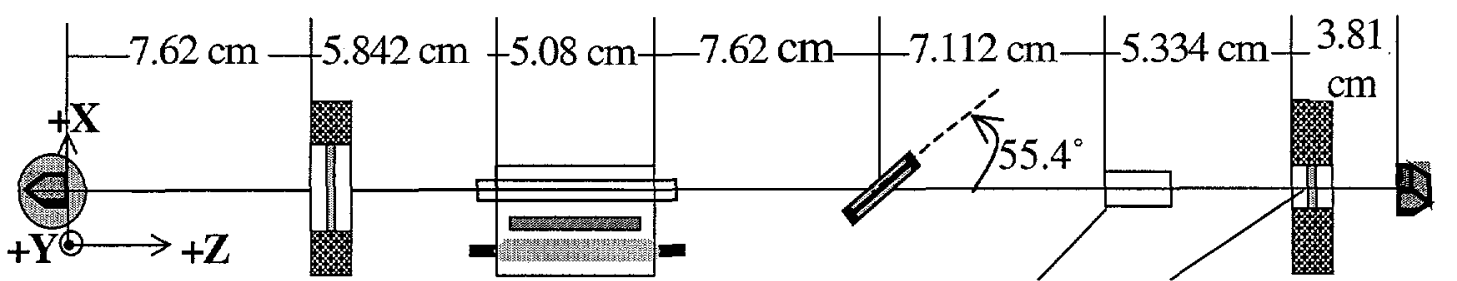

Porro \#1 Waveplate 1 Pump

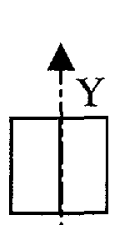

Porro prism 1 view

(Looking at crest along $+Z$ direction)
Chamber
TFP

LiNbO Waveplate 2 Porro\#2

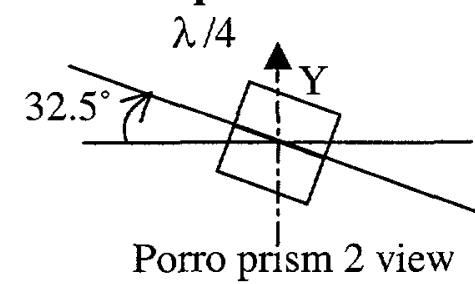

(Looking at crest along $-\mathrm{Z}$ direction)

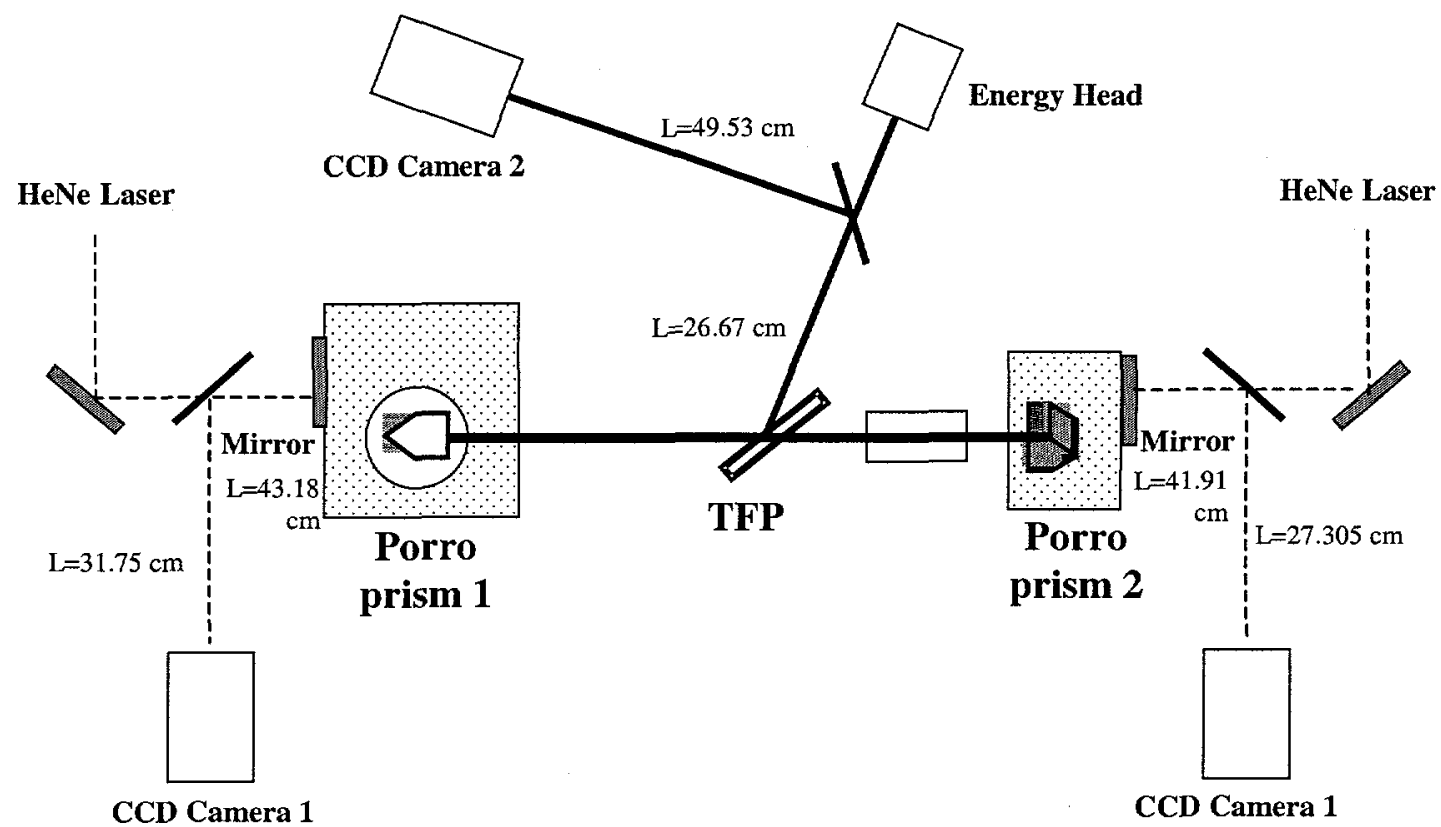

Figure 6 

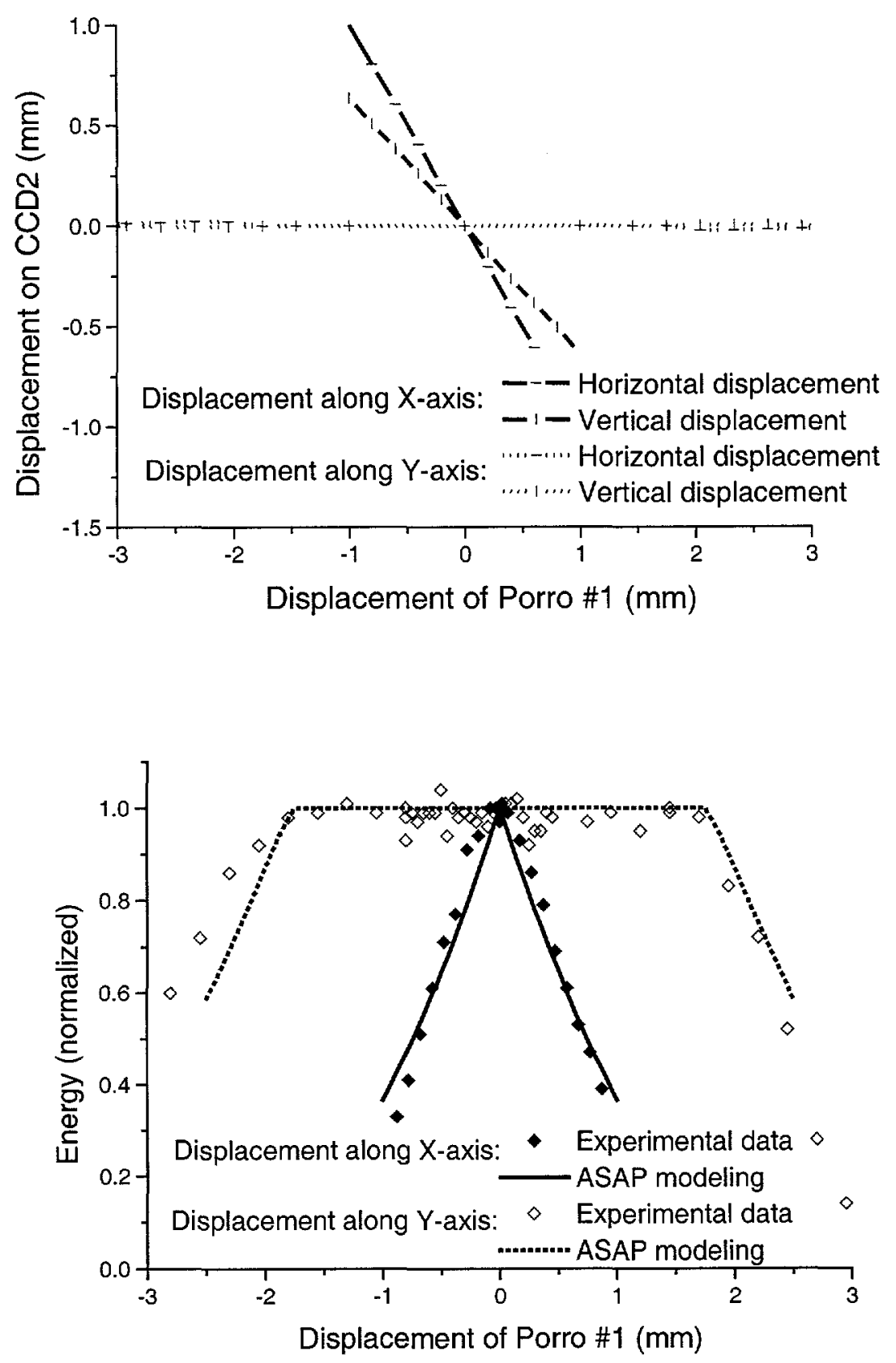

Figure 7 

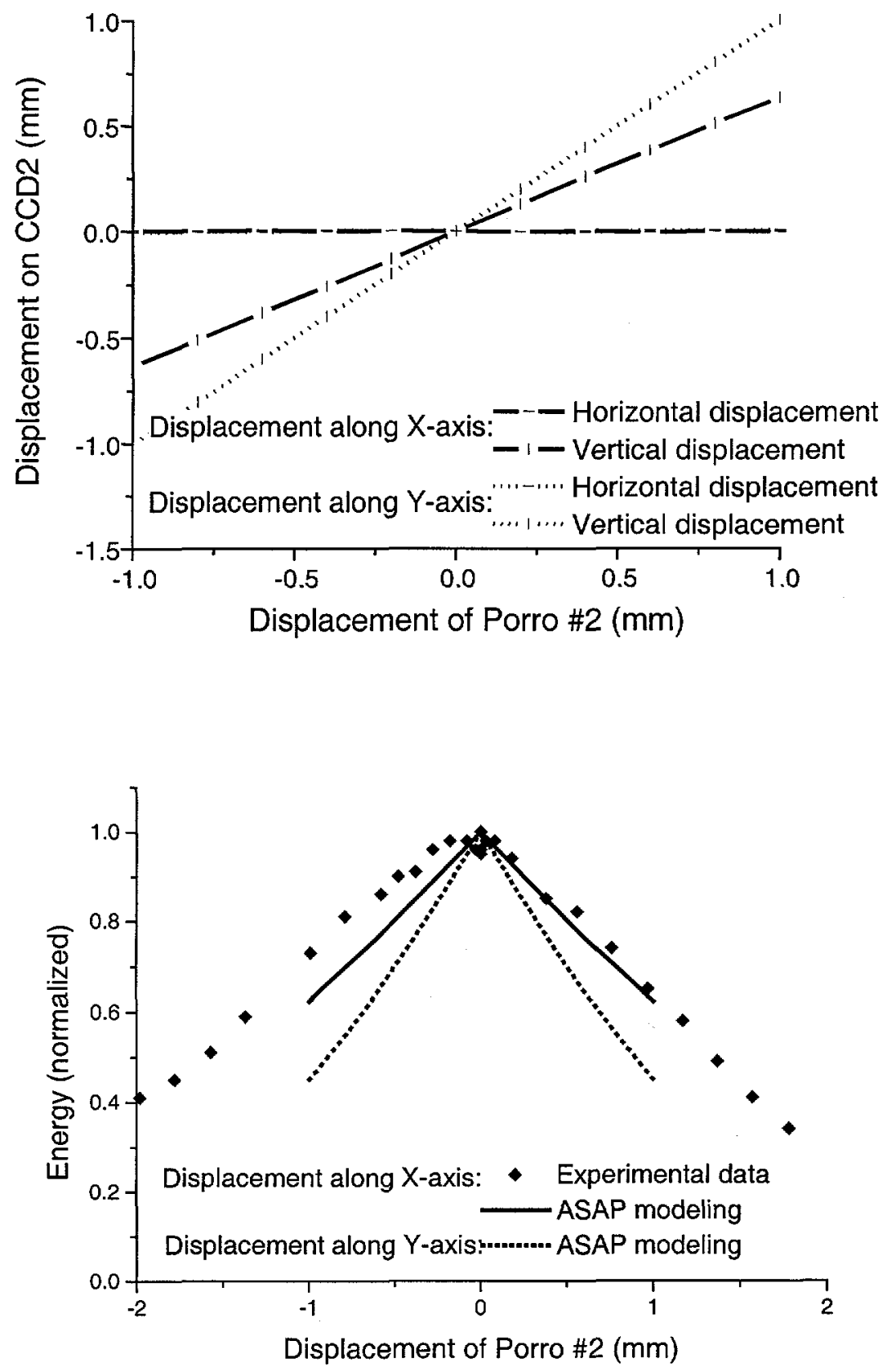

Figure 8 

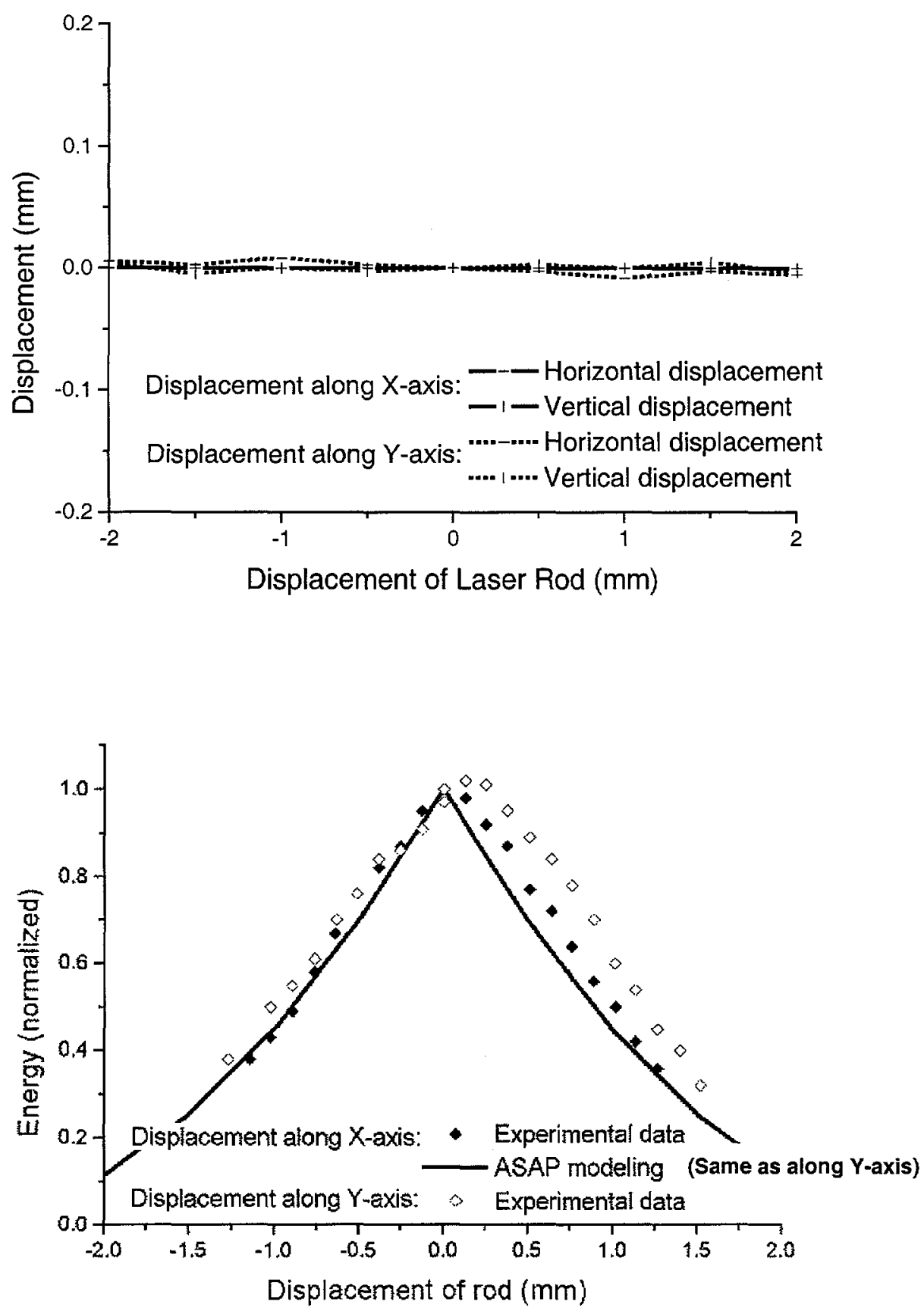

Figure 9 

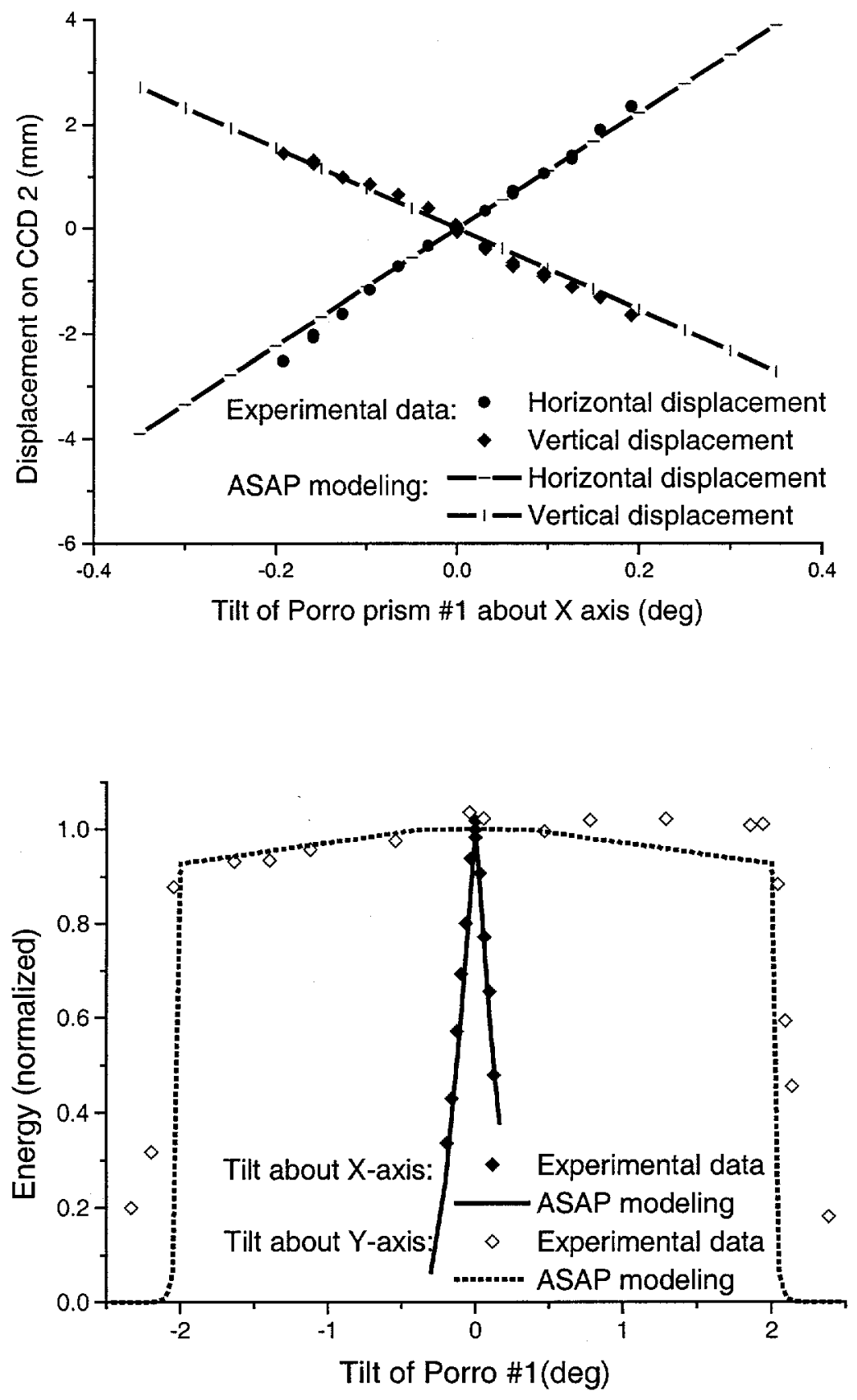

Figure 10 

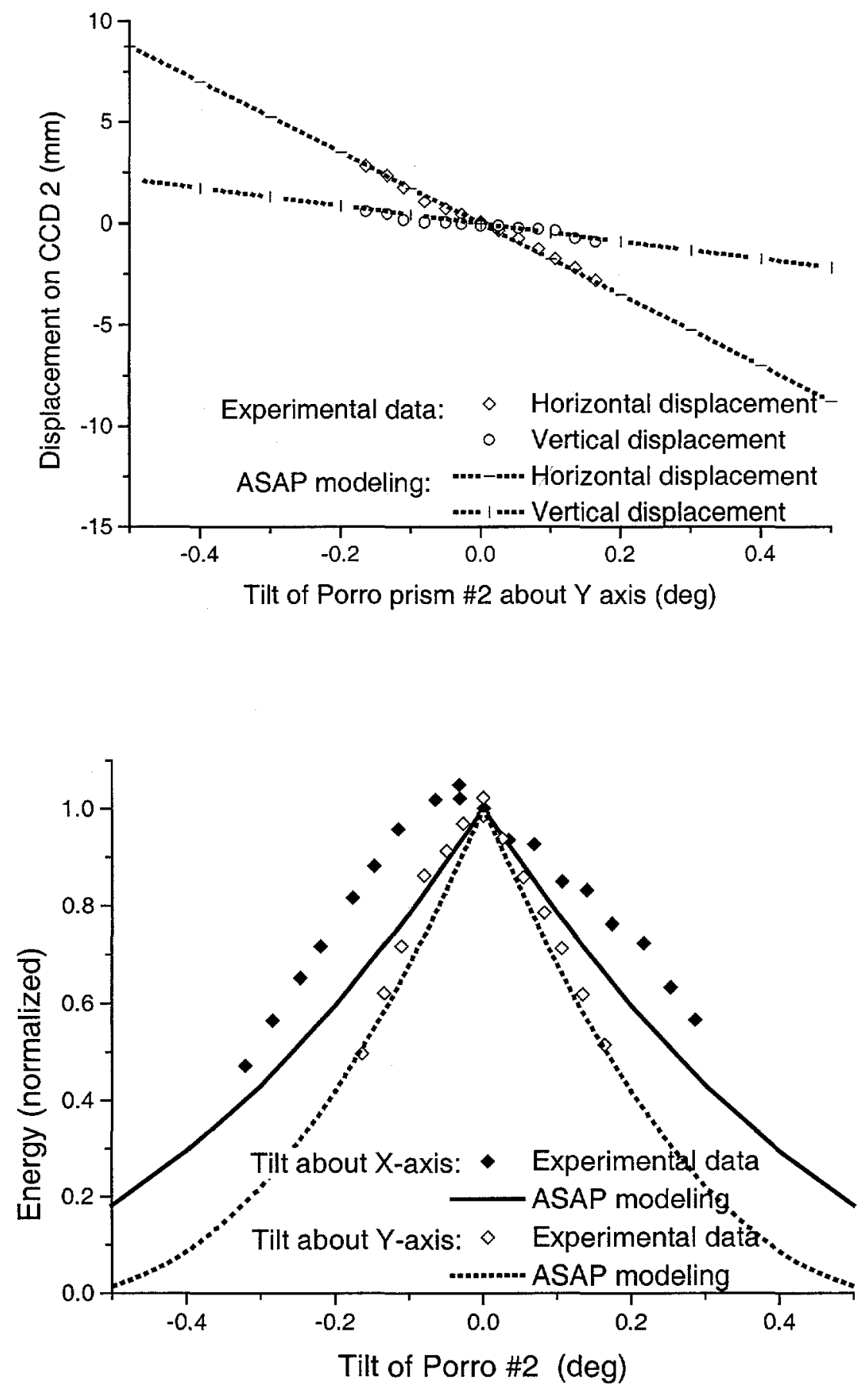

Figure 11 


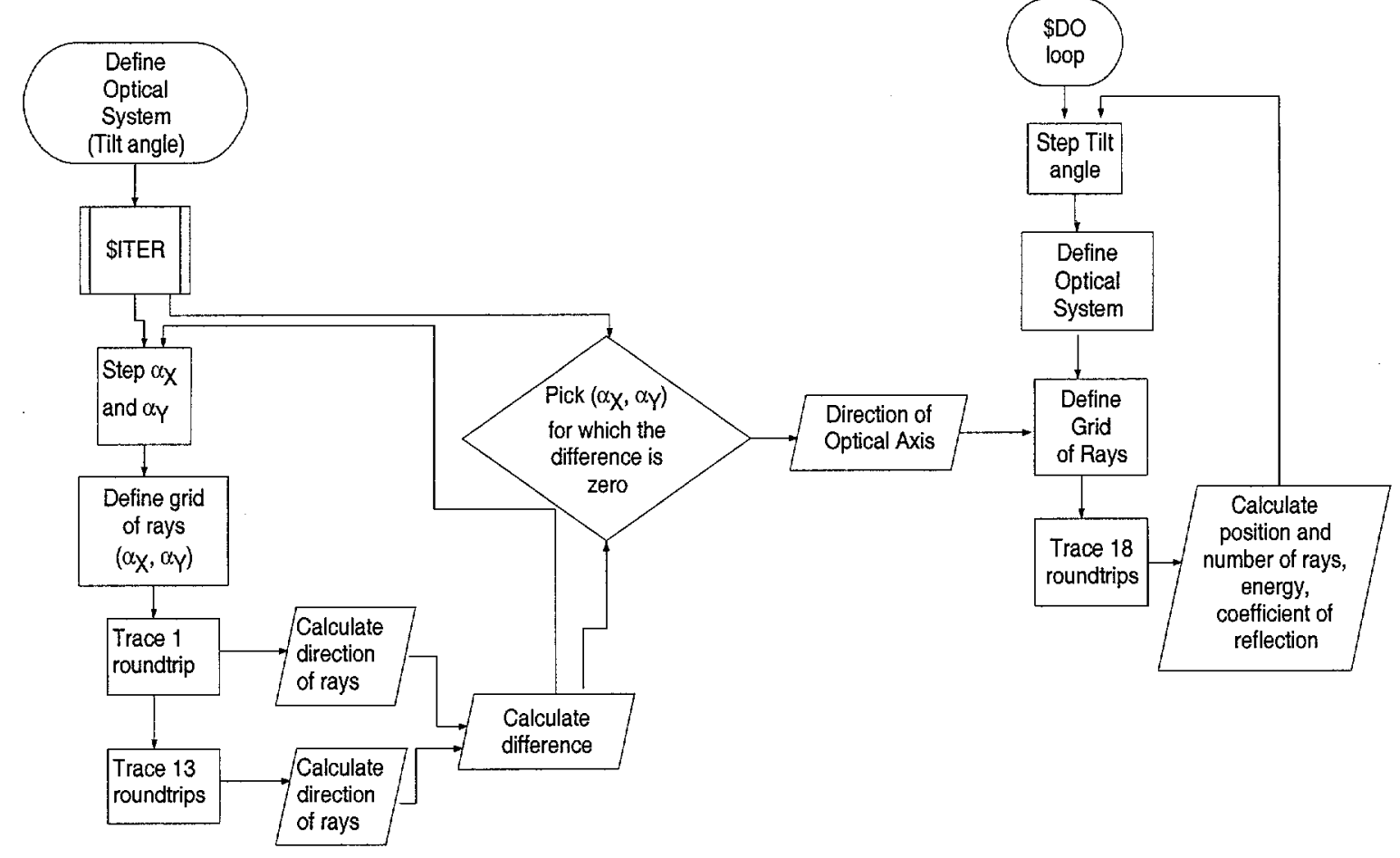

Figure 12 